\title{
One Graph Is Worth a Thousand Logs: Uncovering Hidden Structures in Massive System Event Logs
}

\author{
Michal Aharon, Gilad Barash, Ira Cohen, and Eli Mordechai \\ HP-Labs Israel, Technion City, Haifa, Israel \\ firstname. lastname@hp.com
}

\begin{abstract}
In this paper we describe our work on pattern discovery in system event logs. For discovering the patterns we developed two novel algorithms. The first is a sequential and efficient text clustering algorithm which automatically discovers the templates generating the messages. The second, the PARIS algorithm (Principle Atom Recognition In Sets), is a novel algorithm which discovers patterns of messages that represent processes occurring in the system. We demonstrate the usefulness of our analysis, on real world logs from various systems, for debugging of complex systems, efficient search and visualization of logs and characterization of system behavior.
\end{abstract}

\section{Introduction}

Almost every piece of software writes messages into event logs. Systems composed of many components, such as web services, complex enterprise applications and even complex printing presses collect such events from their many components into log files. These logs are meant to be utilized both in the development stages and in normal operations for debugging and understanding of the behavior of the complex system. In fact, studies have shown these reasons to be the main impetus of companies in collecting logs in the first place [1].

While these logs hold vast amount of information describing the behavior of applications and systems, finding relevant information within the logs can be a very challenging task; even modest systems can log thousands of messages per second. Most users still use the good old unix "grep" for finding potentially relevant messages in event logs. Existing commercial tools, such as those from Splunk, LogLogic and Xpolog, collect and join logs from different sources and provide a more convenient search through the logs. However, the indexing provided by these tools still does not lead to automation in leveraging the logs for tasks such as automated problem debugging, process identification or visualization of the information in the logs.

There are two main fundamental challenges in transforming the logs into machine readable form, enabling automated analysis.

First, a "translation" of the text based events (messages) in the logs into a dictionary of event types: The number of templates creating events is limited, but the actual number of distinct events observed is very large, and grows quickly as a function of time. This is because the actual messages in the logs include various parameters that change from instance to instance of the same type of message (e.g., "login user 
\$name"). Unfortunately, in many systems, the event templates generating the messages are not available, making it challenging to compute representative statistics enabling automated analysis over the events.

Second, a pattern finding mechanism to provide a compressed/concise representation of processes represented in the logs is a major challenge. Many processes spawn multiple messages into logs, e.g., a failure of a process can cause multiple messages in different logs representing the output of different software components creating interleaved sequences of events. Automated systems benefit greatly from identification and representation of such groups, as opposed to individual messages, as it reduces noise, compresses the data and provides more accurate representation of processes in the system.

Solving both of these problems requires the design of efficient machine learning algorithms. Existing research in the area focused mainly on the second problem analysis of log patterns from event log streams [10][11][12][13][14] . These works focus on discovery of temporal patterns or correlation of event statistics to problems, but either assume a known dictionary [10][11][12][13] or assume access to the source code for its discovery [14]. Most of these works generally ignore the complexities introduced when collecting logs from various components in a complex system, namely, interleaving of sequences of events, asynchronous events and high dimensionality.

In this paper we describe our solution to the above challenges and demonstrate the solution on a number of common use cases. Our solution includes two novel algorithms. The first is an efficient sequential text clustering algorithm for creating the dictionary representing the event types. The second, the PARIS algorithm (Principle Atom Recognition In Sets), automatically discovers principle patterns (atoms) of log event types, representing processes in the system. We use the output of these algorithms to visualize logs, enable fast debugging of system problems, and search in the logs via a highly compressed lossless representation of the information in the logs.

The paper is organized as follows. We start with a problem description in the next section. We then describe the two main contributions of this paper; an online dictionary creation algorithm for semi-structured machine generated events, followed by the PARIS algorithm. We discuss the use cases for our log analysis algorithms and demonstrate the results of these algorithms on several large scale datasets collected from enterprise applications. Next, we describe the related work followed by a discussion and conclusions.

\section{Problem Description}

Logs are semi-structured events generated automatically when software components output messages describing actions, warnings or errors during their operation. A log event typically has a timestamp, representing the time at which the software wrote the event, and at least a text message describing the event. In some log events additional fields appear, often describing severity level, source method/function, etc. 
Table 1. Log entry examples from an enterprise application. Marked words represent variables in the templates. Sequences of a similar process are marked 1,2,3 in the first column.
2008-02-06 14:35:16
unexpected failure while trying to ping user session \#55555 the session authentication has failed
2008-02-06 14:35:17
failed to retrieve the meta data of project 'nullo' the session authentication has failed.
2008-02-06 14:35:19
failed to get licenses for project session the session authentication has failed.
2008-02-06 14:35:19
error processing request from 192.111.22.33 data starts with $0 \backslash 00000023 \backslash 0$ conststr download
2008-02-06 14:35:39
unexpected failure while trying to ping user session \#44444 the session authentication has failed
2008-02-06 14:35:40
failed to retrieve the meta data of project 'null1' the session authentication has failed.
2008-02-06 14:35:41
2008-02-06 14:35:41
unexpected failure while trying to ping user session \#33333 the session authentication has failed
2008-02-06 14:50:08
failed to get licenses for project session the session authentication has failed.
2008-02-06 14:50:09
failed to get licenses for project session the session authentication has failed.
2008-02-06 14:50:11
Failed to retrieve the meta data of project 'null3' the session authentication has failed.
2008-02-06 14:50:14
error processing request from 192.111.22.33 data starts with $0 \backslash 00000512 \backslash 0$ conststr download

Consider the examples of log entries from a running application shown in Table 1.

Even though the events in this log contain 10 distinct messages, it is easy to see that there are only four message templates that generated the messages in this log. Variables words (IP address, user session \#, project name, conststr) generated the 10 distinct messages shown. If the templates were known, it would be easy to map each message to its generating templates; however, such templates are rarely known in practice. In addition, the number of log events with distinct messages in the log files we collected represented between $10-70 \%$ of the total number of log events. With millions of log events this number becomes too high for any type of automated analysis on the event log time sequence. The problem then becomes to "compress" the log events so that events with similar messages are grouped together. In this paper we pose this problem as a clustering one, with the goal of recovering the message templates generating the log events.

A second type of behavior is observed in logs when a system reaches a certain state; causing different software components to output log entries, sometimes in an ordered sequence, and sometimes unordered. The log entries in Table 1 show three sequences of $\log$ entries (marked as 1,2,3 in the first column), each caused by the same application state - a failure to authenticate a user session. While some of the event types always occur when an authentication failure occurs (the first three), the fourth message, 'error processing request from ...' occurs in other states as well. It is desirable to capture such processes and represent them as one for better characterization of the system behavior. The second problem is thus to automatically discover such event sequences from the massive logs. Clearly, this second problem requires a solution to the first one, so that sequences can be compared and matched. A further challenge in solving this problem stems from the fact that the logs entries may represent multiple system states at the same time, leading to interleaved sequences (e.g., sequence 2 and 3 above), each representing the same or a different state; e.g., a user session authentication failure may occur at the same time as a failure to update a database - the logs would represent interleaved events from the two failures, without clear 
indication of the source of the failure. Any solution must be capable of discovering the different sequences despite the interleaving.

Formally, we represent each log entry, e, by the tuple (t,msg), where $t$ is the timestamp of the message and msg is the message text, represented by the word vector: $\mathrm{msg}=\mathrm{w}_{1}, \mathrm{w}_{2}, \ldots, \mathrm{w}_{\mathrm{n}}$, where $\mathrm{w}_{\mathrm{i}}$ is the word in the $\mathrm{i}$ 'th position, while $\mathrm{n}$ is the number of words in a message. Each $\mathrm{w}_{\mathrm{i}}$ represents a word from all the words present in the logs, and the set of log entries is E. The first problem is to discover a set of message clusters $\mathrm{C}=\mathrm{c}_{1}, \mathrm{c}_{2}, \mathrm{c}_{3}, \ldots, \mathrm{c}_{\mathrm{k}}$, where $\mathrm{k}<<|\mathrm{E}|$, and map each event $\mathrm{e}(\mathrm{t}, \mathrm{msg})$ to one of the clusters, leading to the new representation of each event as $\left(t, c_{i}\right)$. The second problem is to automatically discover repeating event sequences, even when interleaving between sequences occurs, and when event clusters appear in multiple different types of sequences.

\section{Online Dictionary Creation Algorithm}

To create the dictionary, mapping the text messages to a typically much smaller set of message clusters, we leverage the fact that messages produced by the same template are usually identical in many of the words, with differences only at various parameters. Additionally, word ordering is important, therefore any similarity function needs to take word ordering into account. In our implementation we used the order sensitive cosine similarity between the messages:

$$
\text { (Eq. 1) }<m s g_{1}, m s g_{2}>=\frac{n_{12}}{\sqrt{n_{1} \cdot n_{2}}},
$$

where $\mathrm{n}_{12}$ is the number of identical words comparing each word position of $\mathrm{msg}_{1}$ and $\mathrm{msg}_{2}$, and $\mathrm{n}_{1}, \mathrm{n}_{2}$ are the number of words in each message.

An alternative to the cosine similarity defined above is an edit distance, or variations of it, allowing for insertions and deletions. However, we found that in practice there is little need for it, and using it adds a significant computational overhead.

Additionally, any algorithm is required to meet the following:

1. Efficient: Given the massive amount of log events, any algorithm must process the logs very quickly, keeping up at least with the rate of incoming messages. Our algorithm is linear time in terms of number of messages. Our implementation of the algorithm is able to process 25,000 messages per second, keeping up with all systems we have encountered so far.

2. Produce value immediately: Our algorithm is an online algorithm, producing new clusters as needed as more messages are observed.

3. Consistency of clusters: Two messages that belonged to the same cluster at time $t$, cannot belong to conflicting clusters at time $t+1$, otherwise it can result in conflicting conclusions depending on t. Our algorithm builds a forest of cluster trees, and ensures that messages are always a part of the same tree in the forest, thus maintaining global consistency.

4. Similar messages with different semantics or frequent parameter values should be in separate clusters: It occurs that two messages are almost identical, but the semantics can be very different - e.g., "network is up" vs. "network is down" (often the message can be much longer, with the only difference being 
the words "up" and "down"). Additionally, a message could be "login user \$name" could have many instances where \$name = "root", and the rest with varying names, implying that user "root" should probably be considered separately from the others. To produce the desired result in such cases, our algorithm splits clusters based on the entropy of word positions in the messages and the words within each position to maintain high entropy among the members of a cluster in the word positions considered parameters.

Our online dictionary creation algorithm is shown in Table 2.

The algorithm begins with an empty set of clusters. Each new event is compared to a representative message of the existing clusters in the order in which the clusters were created, and is assigned to the first cluster to which the similarity threshold is exceeded; this ensures the satisfaction of the consistency requirement. If the similarity threshold is not surpassed for any of the existing clusters, a new cluster is created and the event message is used as the representative message of the new cluster.

Table 2. Dictionary creation algorithm

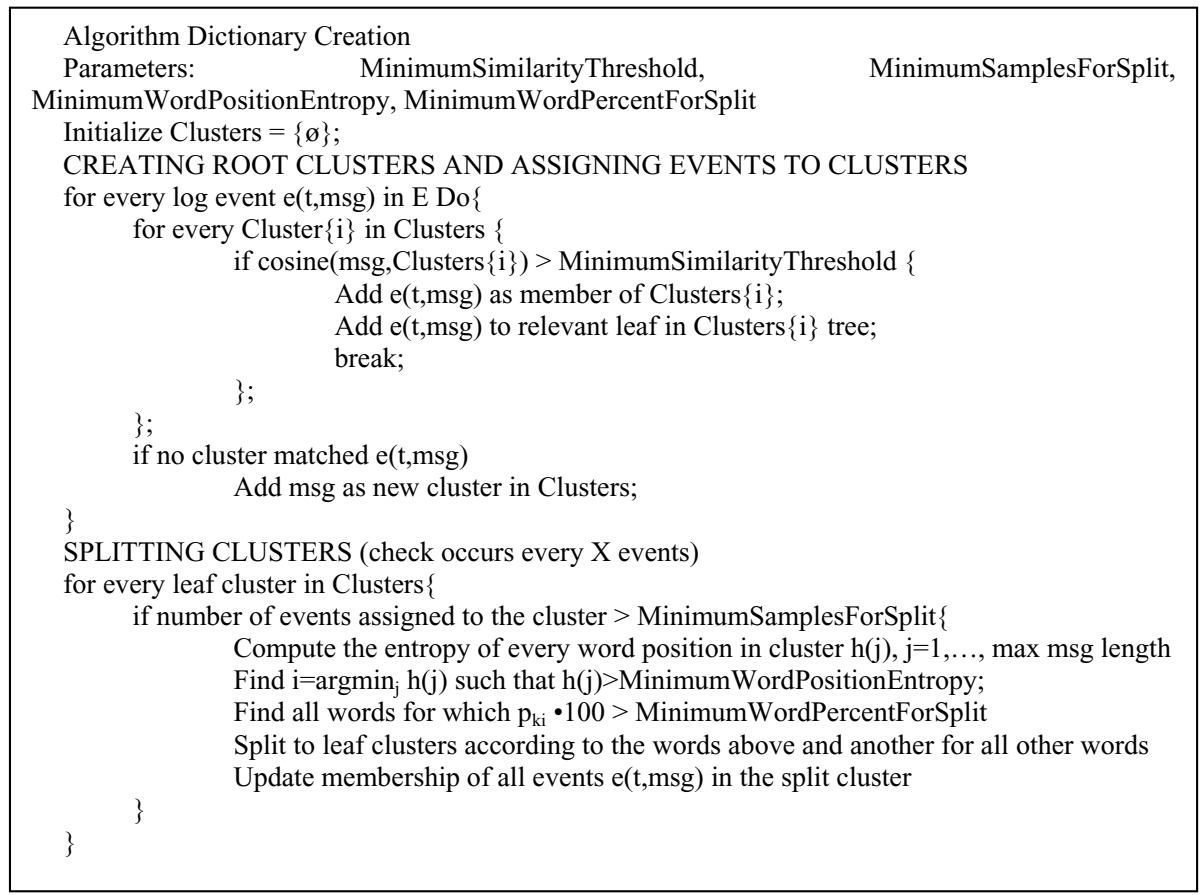

The second step of the algorithm considers splitting a cluster if the following conditions are met:

1. There is a minimum number of events that belonged to the cluster

2. A word position has an entropy smaller than a splitting threshold (but not zero) and at least one word in that word position appeared in $\mathrm{x} \%$ of the messages. 
The entropy of a word position is computed as: $h(j)=-\sum_{k=1}^{n} p_{k j} \cdot \log \left(p_{k j}\right)$, where $\mathrm{n}$ is the number of words in the dictionary, $\mathrm{p}_{\mathrm{kj}}$ is the probability that word $\mathrm{k}$ appears in position $\mathrm{j}$, computed as $p_{k j}=n_{k j} / n_{c}$, where $\mathrm{n}_{\mathrm{kj}}$ is the number of times word $\mathrm{k}$ appeared in position $\mathrm{j}$, and $\mathrm{n}_{\mathrm{c}}$ is the number of messages belonging to the cluster.

When the two conditions are met, a cluster is split into at least two clusters, and possibly more if more words that pass the $\mathrm{x} \%$ threshold. We use $\mathrm{x}=10 \%$ in our experiment, and the minimum number of messages in a cluster as 1000 .

The output of the algorithm is a forest of cluster trees, in which the branches of the tree represent splits based on the entropy criterion, and the tree roots are based on the cosine similarity criterion. In terms of efficiency, the algorithm performs a single pass over the data, preserving word counts for splits as it reads the messages. Creating the root of the forest is purely online, while the splitting phase is performed periodically on select clusters such that new messages are not held up for long. We show in our experiments that despite the heuristic nature of the algorithm, it achieves clustering results that are both accurate in terms of recovering message templates, and also very similar to batch clustering on the same data.

\section{PARIS Algorithm}

The PARIS (for Principal Atoms Recognition In Sets) algorithm is designed for identification of sets of events that tend to occur together. We assume that in each point in time several processes occur in the system, each generates its own set of log messages and the full $\log$ is a union of the individual log sets. We also do not assume that the messages generated by a process are ordered since many systems are a-synchronic making the ordering meaningless. PARIS gets as input the full log, and identifies the individual sets of messages that belong to one process or failure. Therefore, PARIS actually provides an alternative representation of the full $\log$ as a collection of atoms, where each atom is a known set of messages produced by one process or failure. Such an alternative representation provides several advantages,

1. Event suppression and filtering - one atom is used instead a set of log messages, which allows efficient representation of the data.

2. Analysis - each atom stands for one process or failure, and therefore representation by atoms is more meaningful than the full representation. Note that unlike atoms, a single log message can appear in several different processes or failures, and therefore its occurrence does not necessarily describe any specific system state.

3. Reduction of noise - lets assume a process occurs every once in a while in the system, and causes a set of log messages. PARIS will identify this set as an atom that describes the process. Let's also assume in a new time window we witness most of these log messages but not all of them. This inconsistency can be either noise (other messages were deleted, delayed or were wrongly assigned to a different time window), or as an intrinsic problem in the system 
(e.g. the process was stopped in the middle). In both cases, PARIS representation can easily detect this inconsistency - fix it, or alert.

\section{PARIS - Problem Description}

Let $\mathrm{D}_{1}, \mathrm{D}_{2}, \ldots \mathrm{D}_{\mathrm{N}}$ be $\mathrm{N}$ different sets of elements, each consists of a finite set of values taken from a finite alphabet $\mathrm{v}_{1}, \mathrm{v}_{2}, \ldots, \mathrm{v}_{\mathrm{T}}$ (in our case the alphabet is the set of log messages ids, given as a result of the dictionary creation algorithm). We assume the content of each set $\mathrm{D}_{\mathrm{i}}$ consists of at most $\mathrm{L}$ smaller sets, denoted also as principal atoms. The set of all principal atoms is denoted as $A$, and consists of $K$ elements $A=\left\{A_{1}\right.$, $\left.A_{2}, \ldots, A_{K}\right\}$. Each principal atom $A_{j}$ holds a set of values $A_{j}=\left\{v_{j 1}, v_{j 2}, \ldots, v_{j w}\right\}$. The atoms are not necessarily distinct, nor do they consist of all available values. A representation of the set $\mathrm{D}_{\mathrm{i}}$ using $\mathrm{A}$ is denoted as $\mathrm{F}\left(\mathrm{A}, \mathrm{R}_{\mathrm{i}}\right)$, where $\mathrm{R}_{\mathrm{i}}$ is a set of indices, and $F\left(A, R_{i}\right)=\cup_{j \in R_{i}} A_{j}$. We denote by $\mathrm{F}(\mathrm{A}, \mathrm{R})$ the set of all representations, $F(A, R)=\left\{F\left(A, R_{i}\right)|1 \leq i \leq| R \mid\right\}$. The PARIS algorithm aims to find a set of atoms $\mathrm{A}$ and a set of representations $\mathrm{R}$ that minimize the sum of distances between $F\left(A, R_{i}\right)$ and $\mathrm{D}_{\mathrm{i}}$ for $1 \leq i \leq N$. Therefor, the cost function can be defined as

(Eq 2) $\{A, R\}=\underset{A, R}{\arg \min } \sum_{i=1}^{N} d\left(D_{i}, F\left(A, R_{i}\right)\right)$ s.t. $\forall_{i}\left|R_{i}\right| \leq L,|A| \leq k$,

where $d$ is a distance metric between sets. PARIS's execution and results heavily depend on the definition of the distance function $d$. The simplest distance function we defined counts the number of elements that are not common in the two sets, and normalizes it by the size of $D_{i}$,

$$
d\left(D_{i}, F\left(A, R_{i}\right)\right)=\frac{\left|D_{i} \otimes F\left(A, R_{i}\right)\right|}{\left|D_{i}\right|},
$$

where $\otimes$ is the XOR operator that returns the set of elements that appear in one set and not in the other. However, in many cases we found this distance function to be too simplified, as it does not consider cases in which only part of the atom appears in $\mathrm{D}_{\mathrm{i}}$ due to, for example, missing log entries. We defined a slightly modified distance function that uses a slack parameter $r$,

$$
d^{r}\left(D_{i}, F\left(A, R_{i}\right)\right)=d\left(D_{i}, B\left(F\left(A, R_{i}\right), D_{i}, r\right)\right),
$$

Where

$$
B\left(F\left(A, R_{i}\right), D_{i}, r\right)=F\left(\tilde{A}, R_{i}\right),
$$

and $\widetilde{A}_{i}=\underset{\tilde{A}}{\arg \min } d\left(D_{i}, F\left(\widetilde{A}, R_{i}\right)\right)$ for $\widetilde{A}_{i} \subseteq A_{i},\left|\tilde{A}_{i}\right| \geq r \cdot\left|A_{i}\right|$. That is, we allow the distance metric to consider only a portion $r$ of the elements of each atom in the representation reducing the penalty for mismatches. When $r=1$, the function $d^{r}$ is identical to $d$. 


\section{Implementing PARIS}

As the solution of (2) is combinatorial in its nature, we designed an iterative scheme for minimizing it. In each iteration there are two stages: representation stage and atom optimization stage.

- Representation stage: we fix the set $\mathbf{A}$, and solve for all $\mathrm{i}$ $R_{i}=\arg \min d^{r}\left(D_{i}, F\left(A, R_{i}\right)\right)$. This is done greedily by adding one atom $R_{i}$

after the other to the representation. In each stage the added atom is the one that best minimizes the distance. The process stops when the distance is no longer minimized, or when $\left|R_{i}\right|=L$.

- Atom optimization stage: we change A in order to minimize the distance of the representation to $\mathrm{D}$. We do so one atom after the other. When optimizing $A_{i}$, we fix all other atoms, and consider only the data sets that include $A_{i}$ in their representation (other data sets will not effect $A_{i}$ ). For each such data set we define representation error set $\mathrm{E}_{\mathrm{i}}$,

$$
E_{i}=\left\{D_{i} / F\left(A,\left\{R_{i} / i\right\}\right)\right\},
$$

where the operator ' $/$ stands for set subtraction. $E_{\mathrm{i}}$ actually holds all the elements in $D_{i}$ that are not yet represented by all other atoms except $A_{i}$. We then re-define $A_{i}$ in order to better approximate all these representation error sets.

In each iteration the cost function (2) is reduced, and therefore the algorithm is promised to converge to a minimum solution. As the solution is local, several heuristic operations are done in order to force a more global solution (such as replacing identical atoms or atoms that contain other atoms). Other versions or PARIS are designed mainly to omit the need for the initial setting of $\mathrm{K}$ and $\mathrm{L}$, and to better avoid local minima solutions.

\section{Validating PARIS Using Synthetic Data}

A necessary and important stage in validating the algorithm is to execute it on synthetic data that was generated directly according to the model assumptions. For this, we randomly selected a set of K 'true' atoms from an alphabet of size 200 (each atom included 8 elements). Then we assumed a fixed $r$ and generated the input sets $D$. Each set $D_{i}$ was generated by a union of random $L$ atoms. From each of the atoms only $r$ of the elements were taken. In addition, some noise was added to each set by switching on/off $\mathrm{n}$ elements. Finally, we executed the algorithm after supplying it with the parameters $(k, L, r)$, and with the input sets $\mathrm{D}$. The success rate of the algorithm was measured by the number of successful atom restorations out of $\mathrm{k}$.

In the first experiment we set, $\mathrm{K}=50, \mathrm{~L}=3, \mathrm{~N}=3000$, and varying $\mathrm{r}$ (between 0.4 and 1). The rates of successful restorations with noise levels of $0,2,4,10$ are described in the blue, red and green graphs in Figure 1. 


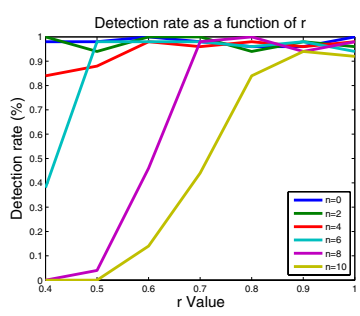

(a)

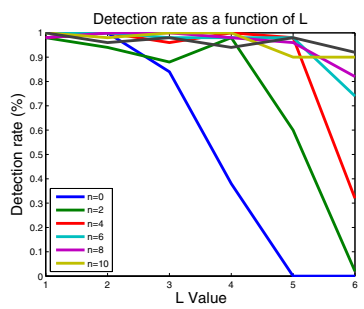

(b)

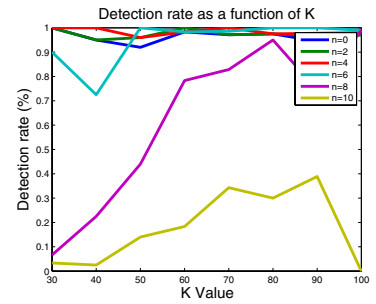

(c)

Fig. 1. (a-c) PARIS synthetic experiments

Another experiment was done with a fixed $\mathrm{r}=0.6$, and $\mathrm{L}$ varies between 1 and 7 , with noise levels of $0,2,4,6,8,10$. The results are presented in Figure 1(b). A third experiment was done with fixed $r=0.6$, and $L=3$, while $K$ varies between 30 and 100 in jumps of 10 and with noise levels of $0,2,4,6,8,10$. The results are presented in Figure 1(c). These results demonstrate the ability of PARIS to successfully extract the original atoms, even in the presence of noise.

However, when applying PARIS on real data, the setting of $\mathrm{K}, \mathrm{L}$ and $\mathrm{r}$ is done heuristically, using reasonable assumptions and knowledge of the system and the origin of the data. In the log experiments we used the following: $k=80, \mathrm{~L}=3, \mathrm{r}=0.51$.

\section{Use Cases for the Log Analysis Algorithms}

The two algorithms described in the previous section transform the system event logs from semi-structured text to machine readable forms. The obvious question is what are the use cases that justify running these algorithms on system logs? In this section we describe three such use cases which we have seen in our experience to be useful for operations of large Enterprise IT systems. There could be additional use cases which we have not encountered yet.

The first use case, and also the most straightforward one, is to use the transformed event $\operatorname{logs}$ to aid in diagnosis of system problems. In most IT applications, indications of problems stem from abnormal measurement values in monitors that are critical to the business, such as transaction response time or throughput. When those indicate a problem, the operators needs to discover the root cause through mounds of data; monitors of system behavior, such as CPU utilization, memory, network, etc, and system event logs. In recent years, work in this area showed how to aid in the diagnosis using machine learning methods such as Bayesian network classifiers, clustering [7][8][9] using real-valued monitors. Adapting these methods to use the output of our algorithm is straightforward; the rate or binary representation of each event type from the dictionary, and/or atoms from the output of PARIS, is computed over time windows of 1-5 minutes, matching the frequency of the monitors used to detect problems. This turns the log events to temporal measurements enabling the application of existing learning technologies to help classify and describe problem periods.

A second use case is visualization of the system event logs over time for gaining better understanding of the system operation. While this use case is similar to the first 
in many respects, visualization of the log events over time produces views that enable quick understanding of system operation, such as reboots, normal periodic processes (e.g., database partition), and processes that are running amok, not causing any detectable problem at the application level yet. Whereas in the first use case the diagnosis of a specific problem that occurred is a supervised learning problem, this use case is unsupervised, leveraging visualization and additional unsupervised techniques to detect anomalies or behavioral patterns from the logs.

A third use case is the use of the output of the algorithm for efficient indexing of the $\operatorname{logs}$, reducing both space requirements and speeding up search through the logs significantly over standard indexing. The clusters serve as the index to each message, coupled with the varying words, to produce a very fast and small index representing exactly all event logs.

We show examples of each use case in the experimental section.

\section{Experimental Results}

For our experiments we collected five log message datasets taken from systems in different fields of the IT world, in accordance with the use cases mentioned above. In order to demonstrate the generality of the algorithm, we chose one hardware log (that of a printing press), one Windows Server event log (which represents an infrastructure environment) and two enterprise business application logs. We were careful to select logs in which system problems were discovered, as well as logs from normal processing time of the systems. We ran each of the logs through the dictionary creation algorithm, transforming them into sets of clusters, unique messages and word dictionaries.

In addition to clustering the messages into distinct clusters, the dictionary creation algorithm also kept track of the distinct message strings, number of distinct nonnumeric words in the logs of each system, as well as statistical information about the messages, such as the average number of words and the median number of words in each message.

Table 3. Result Summary for Log Datasets

\begin{tabular}{|l|l|l|l|l|l|l|l|}
\hline Source & Time Frame & $\begin{array}{l}\text { Number } \\
\text { of } \\
\text { messages }\end{array}$ & $\begin{array}{l}\text { Number } \\
\text { of unique } \\
\text { messages }\end{array}$ & $\begin{array}{l}\text { Number } \\
\text { of clusters }\end{array}$ & $\begin{array}{l}\text { Number of } \\
\text { distinct } \\
\text { words }\end{array}$ & $\begin{array}{l}\text { Median } \\
\text { message } \\
\text { length }\end{array}$ & $\begin{array}{l}\text { Index } \\
\text { reduction }\end{array}$ \\
\hline $\begin{array}{l}\text { Business } \\
\text { App 1 }\end{array}$ & $\begin{array}{l}\text { Start: } \\
\text { 2008-01-10 06:00:01 } \\
\text { End: } \\
\text { 2008-09-03 11:14:16 }\end{array}$ & $4,210,513$ & 153,619 & 4,193 & 112,112 & 25 & $90 \%$ \\
\hline $\begin{array}{l}\text { Printer } \\
\text { Press }\end{array}$ & $\begin{array}{l}\text { Start: } \\
\text { 2008-04-14 22:09:10 } \\
\text { End: } \\
2008-04-1422: 12: 51\end{array}$ & 11,204 & 5,631 & 204 & 1,796 & 10 & $50 \%$ \\
\hline $\begin{array}{l}\text { Window } \\
\text { s Events }\end{array}$ & $\begin{array}{l}\text { Start: } \\
2006-09-0501: 55: 19 \\
\text { End: } \\
2009-06-1902: 38: 45\end{array}$ & 66,102 & 25,340 & 476 & 14,550 & 26 & \\
\hline $\begin{array}{l}\text { Business } \\
\text { App 2 }\end{array}$ & $\begin{array}{l}\text { Start: } \\
2009-01-25 ~ 13: 19: 31 \\
\text { End: } \\
2009-03-2309: 06: 33\end{array}$ & 483,768 & 70,102 & 1,115 & 42,057 & 10 & \\
\hline
\end{tabular}


Table 3 summarizes the results of running each of the datasets through the algorithm. For every data set it shows the timeframe of the messages in the log, how many messages were processed (number of messages), how many distinct messages were in the logs (number of unique messages), the number of clusters they were grouped into (number of clusters), the number of distinct words that were discovered in the log (not including numeric strings), and the median number of words in a message.

\section{Index Size Reduction and Space Compression}

Special attention must be given to the last column of Table 3 Result Summary for Log Datasets. As mentioned previously in the third use case, the algorithm efficiently indexes the logs, both reducing space requirements and speeding up search through the logs significantly over standard indexing. The last column of Table 3 demonstrates this. It shows the percentage by which the index size was reduced in the representation of the logs. In the cases of the business applications, the reduction in size was up to $90 \%$ ! This compression in representation of the logs is done on two levels. The first consists of keeping track of unique messages and the number of times they occur rather than keeping track of every instance of every message. The second level of reduction consists of using the cluster as an index to the unique messages, coupled with keeping only the subsequent varying words of every unique pattern message, to produce a very fast and small index representing exactly all event logs. The $90 \%$ reduction is the saving of the clustering step compared to the first step of reduction - an index based on the unique messages. A 99\% reduction is achieved over the naïve index which doesn't keep track of the unique messages.

Figure 2 further demonstrates the reduction in size that the algorithm provides for the Business Application 1 data, showing that over a 6 month period the number of unique messages (labeled as "Distinct Messages") grows at a steep rate while the number of clusters (labeled as "Template Messages") stays relatively very small.

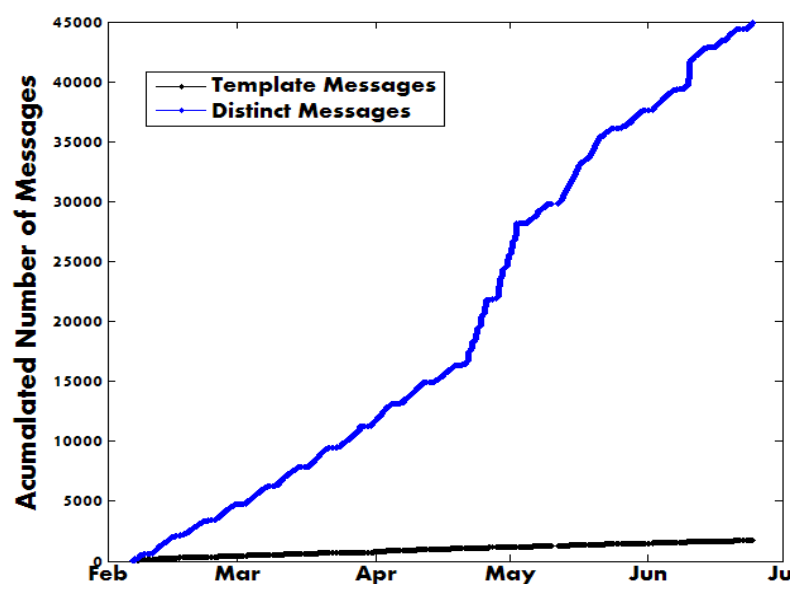

Fig. 2. Growth of distinct (unique) messages vs. cluster (Template) messages created by the algorithm 


\section{Dictionary Accuracy}

In order to gauge the precision of our clustering methodology, we utilized the event logs from Windows to perform an accuracy test of our algorithm. Windows event messages include an event id for every message, a form of ground truth clusters preordained by the Windows server. We analyzed our algorithm's clusters to see what types of Windows event id's are included in each of them, and to see how well our cluster ids correspond to the Windows event id designated by the Windows server. We measure cluster accuracy using clustering purity and normalized mutual information (NMI) [15] compared to the ground truth given by the windows event ids.

For the 25,340 unique messages in the Windows logs, there were a total of 104 distinct event ids and a total of 467 clusters created by the algorithm ${ }^{1}$. The algorithm clustered the events with $96.7 \%$ purity in comparison to the Windows event ids, and NMI of 0.41 . These numbers show that there was a great match between the clusters and the windows event ids, although the algorithm produced four times as many event types as existed in the data. Compared to the number of unique messages though (over 25,000), this number is quite small. It is also noteworthy that some Windows events had different event ids despite being comprised of the exact same text. This ambiguity in the source data can also contribute to the lack of purity in the clusters.

We also compared our clustering algorithm to the result of a batch clustering algorithm on the windows event data. As a batch clustering algorithm we used the hierarchical cluster tree algorithm with single linkage clustering [15], adjusting it to produce the same number of clusters as our online algorithm. The NMI between the results of our clustering ands the batch algorithm was 0.88 , while the purity was at $98 \%$, indicating the result of our online heuristic algorithm is nearly the same as the batch algorithm.

\section{Example 1: Root cause analysis for application performance debugging}

The following example demonstrates the use case of using the log analysis algorithm for diagnosis of performance problems in a transactional business application using the system logs. During routine monitoring of performance, a spike in transaction response time was noticed (see figure 3 ).

In addition, the performance monitors showed that the database CPU usage and the application server CPU usage were increasing, despite the fact that the number of connections to the application server was decreasing.

The monitor indicated multiple symptoms of a performance problem occurring in the system, but no indication of what might be causing it. The different components that compose the environment all have corresponding logs with error messages that occurred at the time of the spike, but no clear way to disseminate them. It is only when processing the system logs through the algorithms that a clear picture of the environment is composed using that information and error messages from multiple sources are put into context of timeline and order (see figure 4).

Once processed through the algorithms, error messages from multiple sources were visualized together, indicating order of appearance and cluster classification.

\footnotetext{
${ }^{1}$ The algorithm ran with similarity threshold of 0.6 .
} 


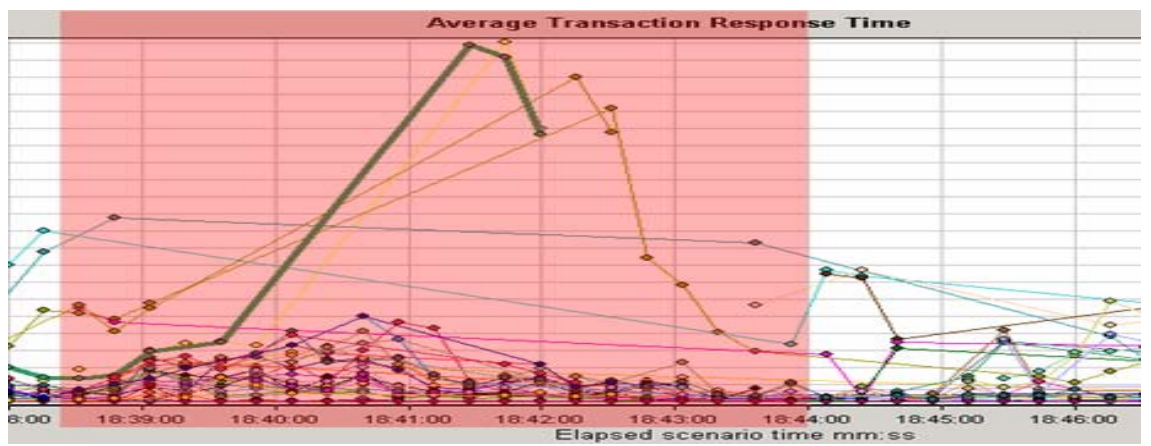

Fig. 3. Spike in transaction response time. The spike represents a performance problem for the application.

Analyzing the messages in this fashion assisted in isolating the root problem that caused the system errors - a java IllegalStateException message: java.lang.IllegalStateException: getAttribute: Session already invalidated. Once identified, explanations and solutions were easily found and applied (e.g., http://forums.sun.com/thread.jspa?threadID=5129793).

\section{Example 2: Error/Behavior Detection in IT Management Software}

The second example we provide is that of an IT Management system whose dashboard did not report any data about system health, despite the data being collected. As opposed to the previous case, in this situation there were no accompanying symptomatic behaviors by components in the system.

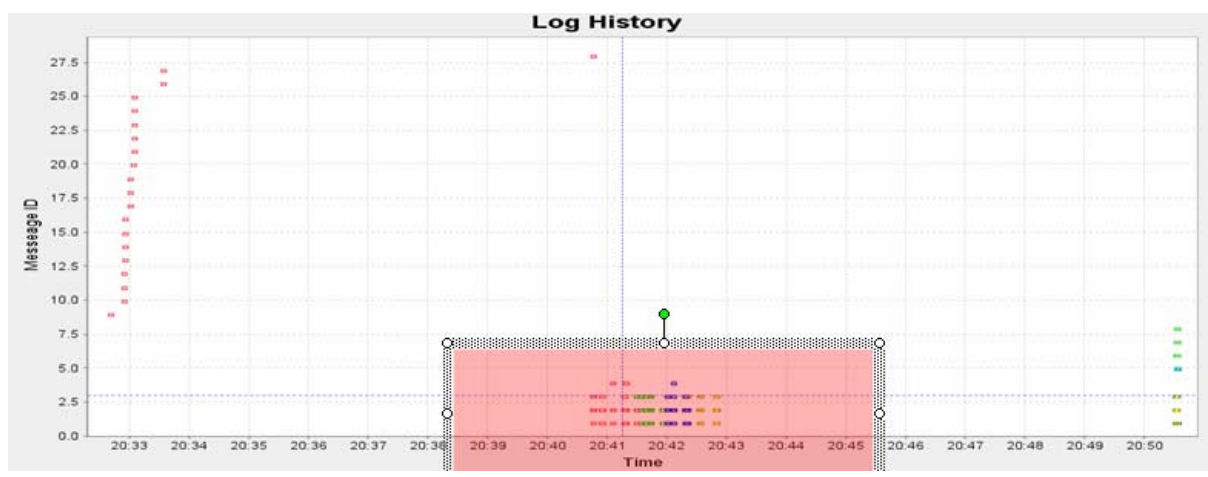

Fig. 4. Error messages at time of system errors, visualized after dictionary creation processing. The $y$-axis refers to the IDs generated by the dictionary creation algorithm (clusters). The $x$ axis is the time. Every dot in the graph represents an actual message from the log, showing its mapping to one of the discovered clusters. The period shaded period corresponds to the period with the spike in response time. The corresponding log error messages relevant to the problem are clearly visible in the graph. 


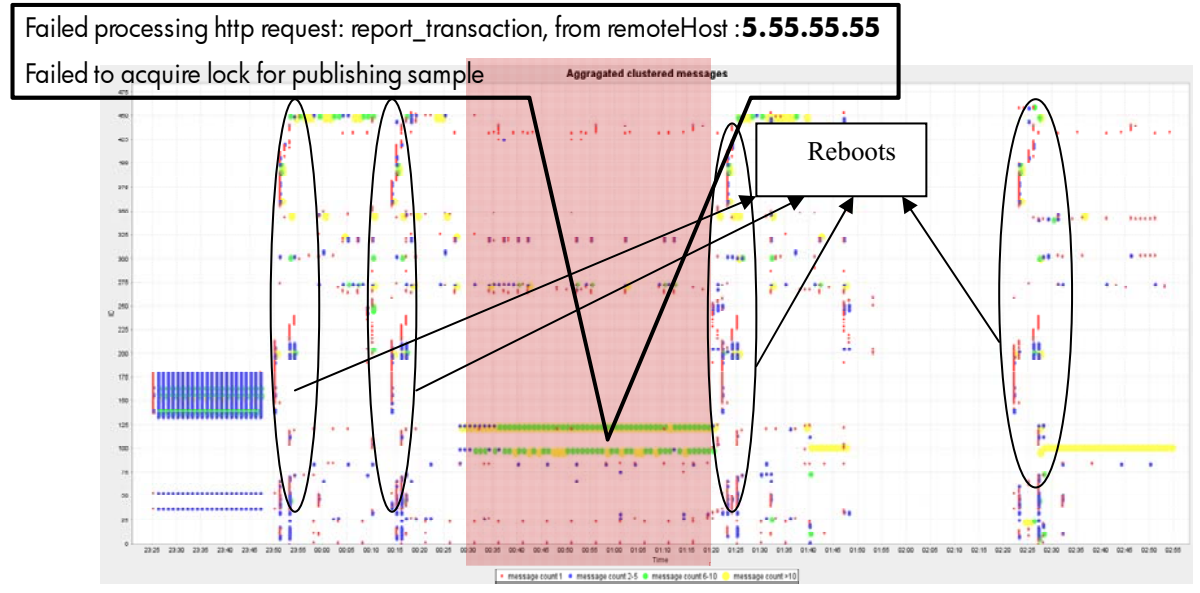

Fig. 5. Visualization of the messages from IT Management System logs following the dictionary creation step. The Region marked in red is the time when a problem occurred in the system; the marked message clusters that appear in this period were deemed to describe the root cause of the problem. Regions marked with ovals demonstrate the pattern of events when the system was performing a restart.

Once again a multitude of logs were processed by the algorithms and, when visualized, provided some insight into the problem in the system. The time-series graph in Figure 5 displays the results of the log analysis process. The area shaded in red is the timeframe in which the problem with application occurred, and upon examination of the graph, the problem is isolated given the two error messages, which appear only during this problem, stating that the report transaction failed to publish its results to the dashboard due to a locking problem. Once again, once the problem is isolated, the corresponding resources can get to work on fixing it.

From the visualization, further information about the system's behavior can be obtained, both normal and abnormal. For example, the points surrounded by ellipses represent groups of messages that recur regularly, which upon investigation are identified as belonging to the normal shutdown and startup processes of the server.

\section{PARIS Results: Identifying Processes}

We have shown in the previous section the result of the PARIS algorithm on artificial data. We applied the PARIS algorithm on the Business App 1 data set, using 15 minute windows and representing the data in the window as a binary vector; a vector representing at time window $t$ is a vector of length $\mathrm{N}$ (the dictionary size), with " 1 "s in the indices of event clusters that appeared at least once during the time window. While no complete ground truth exists regarding the different processes in the system, we resort to experts to determine the validity of our output.

Table 4 below shows examples of three atoms, showing the corresponding event clusters in each. These atoms represent three failure types in the system creating the logs. 
We see that there are shared events in Atom 78 and Atom 14 (highlighted), although the failure modes in the system are different in these two cases. The messages in Atom 79 represent a third failure mode, representing the messages occurring after a session authentication failure. When we looked at the timeline of when this atom appeared, we saw it repeating. Some investigation showed that it is due to an old script that was being executed at a regular interval that went undetected for months until our analysis

Viewing the timeline of when the atoms appeared, we saw that some failure types sometimes occur concurrently, due to the concurrent nature of the system, which serves multiple users performing various different tasks at the same time.

Unfortunately, we do not have ground truth data similar to the windows event logs, as such ground truth requires deep knowledge of the applications. However, the anecdotal evidence presented above do validate both the need for an algorithm such as PARIS and that PARIS can produce meaningful results in complex system event logs.

Table 4. Example Atoms corresponding to three failure types in the system

\begin{tabular}{|l|l|l|}
\hline Failure type 1: Atom 78 & Failure Type 2: Atom 14 & Failure Type 3: Atom 79 \\
\hline $\begin{array}{l}\text { failed to get coverage, } \\
\text { failed to get list of entities keys }\end{array}$ & $\begin{array}{l}\text { failed to cover req invalid values in } \\
\text { removecoverage input frec parameter }\end{array}$ & $\begin{array}{l}\text { failed to post common settings the } \\
\text { project session authentication has } \\
\text { failed. }\end{array}$ \\
\hline $\begin{array}{l}\text { failed to complete the action cannot create } \\
\text { new test duplicate test name 'xxx' }\end{array}$ & $\begin{array}{l}\text { failed to complete the action cannot create } \\
\text { new test duplicate test name 'xxx' }\end{array}$ & $\begin{array}{l}\text { failed to retrieve the meta data of } \\
\text { project 'xxx' the project session } \\
\text { authentication has failed }\end{array}$ \\
\hline $\begin{array}{l}\text { failed to get test value unexpected failure } \\
\text { in getvaluepostprocess }\end{array}$ & $\begin{array}{l}\text { failed to get test value unexpected failure } \\
\text { in getvaluepostprocess }\end{array}$ & $\begin{array}{l}\text { failed to get licenses for } \mathrm{xx} \text { project } \\
\text { session the project session } \\
\text { authentication has failed. }\end{array}$ \\
\hline $\begin{array}{l}\text { failed to post design steps values cannot } \\
\text { add new design step to test \#xxx test \#xxx } \\
\text { not found }\end{array}$ & $\begin{array}{l}\text { failed to post design steps values cannot } \\
\text { add new design step to test \#xxx test \#xxx } \\
\text { not found }\end{array}$ & $\begin{array}{l}\text { failed to get the specified common } \\
\text { settings the project session } \\
\text { authentication has failed. }\end{array}$ \\
\hline & $\begin{array}{l}\text { failed to post req, failed to build } \\
\text { hierarchical item descriptor }\end{array}$ & $\begin{array}{l}\text { error creating request from } \mathrm{x} \backslash \mathrm{xxx} \backslash \mathrm{xx} \\
\text { conststr gettestsetvalue, }\end{array}$ \\
\hline & $\begin{array}{l}\text { failed to lock object the project } \\
\text { session authentication has failed. }\end{array}$ \\
\hline
\end{tabular}

\section{Related Work}

Methods for analyzing system log events have gained the attention of machine learning researchers in recent years. Most works assume a known message dictionary and apply time series analysis methods to the logs. In this category, among others, are [11][12][10], who developed methods to mine temporal event patterns, using HMMs, temporal graphs and other temporal models to extract important patterns. These works assume a known dictionary mapping the messages to a finite set of types, and also do not account for interleaved log sequences, which appear in large scale transactional systems.

[13] introduce a method for reducing the dimensionality of large scale logs by using dimensionality reduction and clustering of groups of log events. However, their method is designed to work in support center settings, where a support engineer receives chunks of logs only when the system is known to have failures, and limits the log types collected. Xu et al [14] also analyze logs based on log message groups and 
use PCA to detect anomalous messages in the logs, analyzing logs continuously during operations. Their solution to the dictionary creation problem is to analyze the source code of the monitored applications, identifying possible message templates from the code and validating them in the logs. In contrast, our method assumes no access to the source code, using only the logs themselves to discover the possible templates, making our method applicable to a wider range of systems. Our PARIS algorithm combines the advantages of both the temporal pattern mining approaches and the batch approaches (such as PCA). It does not ignore time in the analysis, but tries to represent efficiently each time window with a set of atoms, representing processes, thus accounting for both groups of messages and interleaving of sequences.

The PARIS algorithm can also be compared to other existing methods in signal processing and machine learning. The simple version of PARIS is the set variation of the overcomplete dictionary learning algorithm K-SVD [2] in signal processing with real-valued data. K-SVD discovers a dictionary matrix so that each signal in the input data can be well represented by a sparse linear combination of its atoms. Its efficiency in image and video processing was proved in applications such as denoising of images and videos [3][4], image completion [2] and compression [5]. Similar to the latent variables in Latent Dirichlet Allocation [6], PARIS's atoms can be viewed as representing concept clusters, system processes in our case, allowing multiple concept clusters to represent each time period; this property overcomes the interleaving problem present in the logs.

\section{Discussion and Summary}

In this paper we introduced two novel methods for extracting patterns and summarizing complex system event logs. While our work shows promise on real logs, there are various open issues and future work opportunities.

While our dictionary creation algorithm is online and efficient, in some systems it may become necessary to analyze logs locally on the various distributed system machines as it may be too expensive to transfer logs over the network to a single central node. The challenge becomes to generate one consistent dictionary for all logs being analyzed on various machines, so that logs of the same type (e.g., Apache logs from various web servers) produce a single dictionary.

Second, our use of the PARIS algorithm on the logs currently uses a fixed size time window. However, for some processes, shorter or longer windows may be required, and the boundaries of the current windows are arbitrary and may split processes. An adaptive windowing approach may be required for more accurate process identification. Additionally, further validation is required for the PARIS algorithm on logs with more ground truth data.

Our heuristic dictionary creation algorithm performed well on system event logs and would most likely fair well on texts generated automatically from a finite set of templates. While we used the word order sensitive cosine similarity, it can be easily modified to ignore word order and use other similarity measures. We have also been experimenting with the PARIS algorithm to extracts concept classes from general text corpus, with initial results showing promise. 
To conclude, the title of this paper, "one graph is worth a thousand logs", was coined by the quality engineer who used our system to quickly diagnose a performance problem with his application. This paper provides the necessary machine learning methods that transform massive amounts of system event logs into a form that enables meaningful visualizations and automated analysis that indeed help operators understand the complex behavior of the systems they manage.

\section{References}

[1] Shenk, J.: Demanding More from Log Management Systems, A SANS Whitepaper (June 2008),

http: / /www.sans.org/reading_room/analysts_program/ LogMgt_June08.pdf

[2] Aharon, M., Elad, M., Bruckstein, A.M.: The K-SVD: An Algorithm for Designing of Overcomplete Dictionaries for Sparse Representation. The IEEE Trans. On Signal Processing 54(11), 4311-4322 (2006)

[3] Elad, M., Aharon, M.: Image Denoising Via Sparse and Redundant representations over Learned Dictionaries. The IEEE Trans. on Image Processing 15(12), 3736-3745 (2006)

[4] Protter, M., Elad, M.: Image Sequence Denoising Via Sparse and Redundant Representations. IEEE Trans. on Image Processing 18(1), 27-36 (2009)

[5] Bryt, O., Elad, M.: Compression of Facial Images Using the K-SVD Algorithm. Journal of Visual Communication and Image Representation 19(4), 270-283 (2008)

[6] Blei, D.M., Ng, A.Y., Jordan, M.I.: Latent dirichlet allocation. Journal of machine Learning Research 3 (2003)

[7] Cohen, I., Goldszmidt, M., Kelly, T., Symons, J., Chase, J.S.: Correlating instrumentation data to system states: A building block for automated diagnosis and control. In: Proc. 6th USENIX OSDI, San Francisco, CA (December 2004)

[8] Cohen, I., Zhang, S., Goldszmidt, M., Symons, J., Kelly, T., Fox, A.: Capturing, indexing, clustering, and retrieving system history. In: Proc. 20th ACM SOSP (2005)

[9] Powers, R., Cohen, I., Goldszmidt, M.: Short term performance forecasting in enterprise systems. In: SIGKDD 2005 (2005)

[10] Peng, W., Perng, C., Li, T., Wang, H.: Event Summarization for System Management. In: SIGKDD 2007 (2007)

[11] Hellerstein, J.L., Ma, S., Perng, C.-S.: Discovering actionable patterns in event data. IBM System Journal 41(3), 475 (2002)

[12] Li, T., Liang, F., Ma, S., Peng, W.: An integrated framework on mining logs Files for computing system management. In: SIGKDD 2005 (2005)

[13] Sabato, S., Yom-Tov, E., Tsherniak, A., Rossetm, S.: Analyzing System Logs: A New View of What's Important. In: Second Workshop on Tackling Computer Systems Problems with Machine Learning Techniques, SysML 2007 (2007)

[14] Xu, W., Huang, L., Fox, A., Patterson, D., Jordan, M.: Mining Console Logs for LargeScale System Problem Detection. In: SysML 2008 (2008)

[15] Manning, C.D., Raghavan, P., Schütze, H.: Introduction to Information Retrieval. Cambridge University Press, Cambridge (2008) 